\title{
KIR2DS5 in the presence of HLA-C C2 protects against endometriosis
}

\author{
Izabela Nowak • Rafał Ploski • Ewa Barcz • Piotr Dziunycz • Pawel Kamiński • \\ Grażyna Kostrzewa • Lukasz Milewski • Piotr I. Roszkowski • David Senitzer • \\ Jacek Malejczyk • Piotr Kuśnierczyk
}

Received: 15 December 2014 / Accepted: 5 February 2015 / Published online: 1 March 2015

(C) The Author(s) 2015. This article is published with open access at Springerlink.com

\begin{abstract}
Endometriosis is defined as the presence of functional endometrial tissue outside the uterine cavity. Several hypotheses have attempted to explain the etiology and pathogenesis of endometriosis. Recently, it has been suggested that a defect of the natural killer (NK) activity in the recognition and lysis of endometrial cells is one of the crucial points in the
\end{abstract}

Electronic supplementary material The online version of this article (doi:10.1007/s00251-015-0828-3) contains supplementary material, which is available to authorized users.

\footnotetext{
I. Nowak $(\bowtie) \cdot$ P. Kuśnierczyk $(\bowtie)$

Laboratory of Immunogenetics and Tissue Immunology, Department of Clinical Immunology, Ludwik Hirszfeld Institute of Immunology and Experimental Therapy, Polish Academy of

Sciences, ul. Rudolfa Weigla 12, 53-114 Wrocław, Poland

e-mail: izan@iitd.pan.wroc.pl

e-mail: pkusnier@iitd.pan.wroc.pl

R. Płoski $\cdot$ G. Kostrzewa

Department of Medical Genetics, Centre of Biostructure Research, Medical University of Warsaw, Kampus Banacha, ul. Pawińskiego

3c, 02-106 Warszawa, Poland

R. Płoski

e-mail: rploski@wp.pl

G. Kostrzewa

e-mail: grazyna.kostrzewa@wum.edu.pl

E. Barcz $\cdot$ P. Kamiński

First Chair and Clinic of Obstetrics and Gynecology,

Medical University of Warsaw, Pl. Starynkiewcza 1/3,

02-015 Warszawa, Poland

E. Barcz

e-mail: ewa.barcz@interia.pl

P. Kamiński

e-mail: pawel.kaminski@wum.edu.pl
}

development of this disease. Natural killer cells can express killer immunoglobulin-like receptors (KIR), which recognize class I human leukocyte antigens on target cells. We asked whether polymorphisms in KIR, HLA-C, and $H L A-B$ genes are risk factors for endometriosis. We tested 153 women with endometriosis diagnosed on the basis of laparoscopic and

\author{
P. Dziunycz $\cdot$ Ł. Milewski $\cdot$ J. Malejczyk \\ Department of Histology and Embryology, Centre of Biostructure \\ Research, Medical University of Warsaw, ul. Chałubińskiego 5, \\ 02-004 Warsaw, Poland \\ P. Dziunycz \\ e-mail: piotr.dziunycz@gmail.com \\ Ł. Milewski \\ e-mail: lmilewski@o2.pl \\ J. Malejczyk \\ e-mail: jacek.malejczyk@wum.edu.pl
}

\section{P. I. Roszkowski}

Second Clinic of Obstetrics and Gynecology, Medical University of Warsaw, ul. Karowa 2, 00-315 Warszawa, Poland

e-mail: roszkowskip@wp.pl

\author{
D. Senitzer \\ Laboratory of Histocompatibility, City of Hope, National Medical \\ Center, 1500 East Duarte Road, Duarte, CA 91010, USA \\ e-mail: DSenitzer@coh.org
}


histological examination, and 213 control healthy women, who gave birth to at least one child. The frequency of KIR genes in patients was similar to that in controls except for KIR2DS5, which exerted a protective effect only in $H L A-C$ $\mathrm{C} 2$-positive individuals. Moreover, KIR2DS5-positive women with endometriosis had 13 times lower chance that the disease would occupy the peritoneum than KIR2DS5- and KIR2DS4del-negative ones ( $\mathrm{OR}=0.077, P=0.0061)$. Similarly, KIR2DS4del-positive endometriotic persons had 11 times lower chance for peritoneal disease $(\mathrm{OR}=0.094, P<0.001)$. Negative linkage disequilibrium between KIR2DS5 and KIR2DS4del indicates that these genes are mutually exclusive. Our data suggest that KIR2DS5 may be associated with protection from endometriosis, whereas KIR2DS4del seems to be associated with higher disease stages, possibly by exclusion of protective KIR2DS5.

Keywords Endometriosis - Killer cell immunoglobulin-like receptor - Human leukocyte antigen - Linkage disequilibrium

\section{Introduction}

Endometriosis is a common gynecological disease, which affects approximately from 2 to $10 \%$ of women in reproductive age. Patients with endometriosis suffer from pelvic pain, dyspareunia, dysuria, dysmenorrhea, and they constitute about $50 \%$ of infertile women (Dunselman et al. 2014). Endometriosis is defined as the presence of functional endometrial tissue outside the uterine cavity, mostly in the pelvic peritoneum, ovaries, and rectovaginal septum (Burney and Giudice 2012; Gupta et al. 2008; The Practice Committee of the American Society for Reproductive Medicine 2012). Several hypotheses have attempted to explain the etiology and pathogenesis of endometriosis, mainly Sampson's theory of retrograde menstruation of the endometrial tissue through the fallopian tubes and into the peritoneal cavity, and its subsequent implantation (Sampson 1927). It has been suggested that a defect of the natural killer (NK) activity in the recognition and lysis of these implanted endometrial cells may be one of the crucial points in the initiation and progression of endometriosis (Berkkanoglu and Arici 2003).

Natural killer cells can express killer immunoglobulin-like receptors (KIR), which recognize class I human leukocyte antigens (HLA) on target cells and may either stimulate or inhibit NK cell activity. KIR receptors occur as two or three extracellular immunoglobulin-like domain molecules with a long (L) or short (S) cytoplasmic tail. KIR2DL/KIR3DL has inhibitory function in contrast to KIR2DS/3DS with activating function. KIR genes exhibit extensive haplotypic and allelic polymorphism. Individuals differ in both the number and kind (activating vs. inhibitory) of KIR genes (Middleton and Gonzelez 2010; Falco et al. 2013; Kulkarni et al. 2008).

HLA-C molecules can be divided into two groups, HLA-C $\mathrm{C} 1$ and $\mathrm{C} 2$, according to the amino acid present at position 80 of the molecule. All HLA-C molecules with asparagine at position 80 belong to the HLA-C $\mathrm{C} 1$ group, and are recognized by KIR2DL2/2DS2 and KIR2DL3, whereas those with lysine in this position belong to the HLA-C C2 group and constitute ligands for KIR2DL1/2DS1. However, KIR2DL2/ 2DL3 may also bind to some C2 molecules. KIR2DS4 binds HLA-A11 and some $\mathrm{C} 1$ and $\mathrm{C} 2$ molecules (Graef et al. 2009). Finally, KIR3DL1 has specificity for HLA-Bw4 epitope (Middleton and Gonzelez 2010; Falco et al. 2013; Kulkarni et al. 2008).

KIR and HLA polymorphism affects NK cell reactivity and susceptibility to various diseases (Kulkarni et al. 2008). The aim of our study was to find an association of KIR genes and their HLA ligands with susceptibility to endometriosis in Polish women. To our knowledge, this is the first study in females emphasizing the role of KIR2DS5 and KIR2DS4 deletion variant (KIR2DS4del) in clinical stages of endometriosis.

\section{Materials and methods}

Study design

One hundred and fifty-three Polish patients with endometriosis (age range, $20-58$ years; mean \pm S.D., $33.7 \pm 8.0$ years) diagnosed at the First and Second Department of Obstetrics and Gynecology, Medical University of Warsaw for pelvic pain, dysmenorrhea, and/or infertility were included in the study. Patients were classified according to the revised American Fertility Society criteria (American Fertility Society 1985) and both laparoscopic and histopathological examinations. All patients were categorized into four subgroups: III — minimal and mild stages, III—-moderate, and IV—severe stages. Among them, 98 (64.1\%) patients manifested ovarian endometriosis, 43 (28.1\%) patients had ovarian and peritoneal endometriosis, and 12 (7.8\%) patients had only peritoneal endometriosis.

The control group consisted of 213 women. Among this group, 96 women were anonymous adults from paternity testing, who gave birth to at least one child, but there was no information on the disease status as they were anonymous. The remaining 117 women (age range, 23-68 years; mean age \pm S.D., $31.37 \pm 6.36$ ) reported having at least two babies with the same partner and no gynecological, endocrinological, or immunological disorders (described earlier by Nowak et al. 2009). Experimental protocols were approved by the Local Ethics Committee and all patients gave their informed consent to the study. 
DNA preparation and genotyping

Genomic DNA was isolated from venous blood using the salting out method or using an Invisorb Spin Blood Midi Kit (Invitek, Berlin, Germany) following the manufacturer's instructions. KIR genotyping was described previously by Nowak et al. (2009) and Sun et al. (2004). Our KIR typing is validated three times a year by the International KIR Exchange Program organized by the Immunogenetics Center of the University of California at Los Angeles.

$H L A-C$ gene fragments determining the HLA-C1 and $C 2$ groups were distinguished according to a PCR-SSP method described by Frohn et al. (1998). HLA-Bw4 and HLA-Bw6 alleles were genotyped as described in Supplement 1.

Statistical analysis

The proportion of individuals positive for a given $K I R$ gene as well as $H L A-C 1$ and $C 2$ groups and $H L A-B w 4$ was established by direct counting. KIR gene frequencies (gf) were estimated using the formula $\mathrm{gf}=1-\sqrt{ }(1-\mathrm{pf})$, where $\mathrm{pf}$ is the proportion of the population positive for the gene. Odds ratio and its $95 \%$ confidence interval were used as a measure of effect size. Generalized linear models with binomial errors and the chisquare test for trend were used to investigate the relationship between clinical and genetic variables. Akaike's information criterion was used as a measure of fit of models. Confidence intervals were estimated with the bootstrap approach. $P$ values were computed exactly by numerical simulations. Measures for the estimation of linkage disequilibrium (LD) were the correlation of two alleles' frequencies, $r$, global squared correlation between two loci, $R^{2}$ and KullbackLeibler divergence of two loci from LE (Excoffier and Slatkin 1995; Abdallah et al. 2003). For the two loci 2DS4 and 2DS5, $r$ and $R^{2}$ were obtained as: $r=\frac{D_{i j}}{\sqrt{p_{i} q_{j}}}$, where $p_{i}$ and $q_{j}$ are the population allele frequencies of the $i$ th allele on locus 2DS4 and the $j$ th allele on locus $2 D S 5, D_{i j}=x_{i j}-p_{i} q_{j}$, and $x_{i j}$ is the frequency of the haplotype with alleles $i$ and $j$ on loci $2 D S 4$ and $2 D S 5$, respectively. $R^{2}=\sum_{i}^{3} \sum_{j}^{2} \frac{D_{i j}^{2}}{p_{i} q_{j}}$. Kullback-Leibler divergence (Bhasi et al. 2006; Liu and Lin 2005), $D_{\mathrm{KL}}$ is a measure of distance between the observed haplotype distribution and the expected distribution assuming LE: $D_{\mathrm{KL}}=$ $\sum_{i}^{3} \sum_{j}^{2} x_{i j} \log \frac{x_{i j}}{p_{i} q_{j}}$. The chi-square statistic was calculated to test whether all of the $D_{i j}$ 's between 2DS4 and 2DS5 are zeros: $\chi_{d f=2}^{2}=\sum_{i}^{3} \sum_{j}^{2} \frac{n D_{i j}^{2}}{p_{i} q_{j}}$.

\section{Results}

The frequencies of KIR genes in patients were similar to the frequencies observed in control women (Table 1) except for KIR2DS5. This latter result has already been described, but without analysis of other KIR genes (Nowak et al. 2010). The presence of KIR2DS5 significantly decreased the risk of endometriosis. The KIR2DS5 effect was independent from other $K I R$ genes, including KIR2DS4 $(P=0.181)$. However, it was seen only in HLA-C C2-positive individuals: in this group, those who possessed KIR2DS5 had 2.5 lower chance of getting endometriosis than KIR2DS5-negative women (Supplement 2). In contrast, KIR2DS5 presence did not affect the risk of disease in $C 2$-negative individuals (Supplement 2). Noteworthy, $C 2$ presence or absence itself had no relation to disease risk $(P=0.363)$. Similarly, there was no correlation of $H L A-B w 4$ or HLA-Bw6 with disease (data not shown).

We also analyzed the genetics of peritoneal versus ovarian endometriosis (Table 2). KIR2DS5-positive, KIR2DS4delnegative women with endometriosis had 13 times lower chance that the disease would occupy the peritoneum than KIR2DS5- and KIR2DS4del-negative ones (OR=0.077, $P=$ $0.0061)$. The percentage of individuals with peritoneal

Table 1 KIR gene frequencies in patients and controls

\begin{tabular}{|c|c|c|c|c|c|c|c|c|c|c|c|c|c|}
\hline \multirow[t]{2}{*}{ Group } & \multicolumn{13}{|c|}{ KIR gene } \\
\hline & 2DL1 & 2DL2 & $2 \mathrm{DS} 3$ & 2DL3 & 2DS2 & 3DL1 & 3DS1 & 2DL5 & 2DS1 & 2DS4fl & 2DS4del & 2DS4 & 2DS5 \\
\hline Patients, $N$ & 147 & 89 & 61 & 135 & 91 & 147 & 51 & 79 & 60 & 51 & 125 & 147 & $33^{*}$ \\
\hline $\mathrm{gf}^{\mathrm{a}}$ & 80.3 & 35.3 & 22.5 & 65.6 & 36.4 & 80.3 & 18.3 & 30.4 & 22.0 & 18.3 & 57.2 & 80.3 & 11.5 \\
\hline Controls, $N$ & 205 & 115 & 70 & 188 & 116 & 201 & 85 & 113 & 94 & 61 & 181 & 201 & 68 \\
\hline gf & 80.5 & 32.2 & 18.1 & 65.8 & 32.5 & 76.3 & 22.5 & 31.4 & 25.2 & 15.5 & 61.3 & 76.3 & 17.5 \\
\hline OR & 0.96 & 1.19 & 1.35 & 1.0 & 1.23 & 1.46 & 0.75 & 0.94 & 0.82 & 1.25 & 0.79 & 1.46 & 0.58 \\
\hline
\end{tabular}

$N$ number of cases, $p$ probability, $O R$ odds ratio, $95 \% C I$ confidence interval

Patients vs controls, $* P=0.033,95 \% \mathrm{CI}=0.36-0.95$

${ }^{\mathrm{a}} \mathrm{gf}$, gene frequency calculated according to the formula $\mathrm{gf}=1-\sqrt{ }(1-\mathrm{pf})$ 
Table 2 Chance of peritoneal endometriosis depending on KIR2DS5/2DS4del genotype

\begin{tabular}{|c|c|c|c|c|c|c|c|c|c|}
\hline \multirow[t]{2}{*}{ 2DS5 } & \multirow[t]{2}{*}{ 2DS4del } & \multicolumn{2}{|c|}{ Peritoneum } & \multirow[t]{2}{*}{ Number } & \multirow[t]{2}{*}{ Percent } & \multirow[t]{2}{*}{ OR } & \multirow[t]{2}{*}{$95 \% \mathrm{CI}$} & & \multirow[t]{2}{*}{$P$ value } \\
\hline & & + & - & & & & & & \\
\hline- & - & 13 & 3 & 16 & 81.25 & 1 & - & - & - \\
\hline+ & + & 9 & 12 & 21 & 42.86 & 0.173 & 0.038 & 0.795 & 0.041 \\
\hline+ & - & 3 & 9 & 12 & 25.00 & 0.077 & 0.012 & 0.478 & 0.0061 \\
\hline- & + & 30 & 74 & 104 & 28.85 & 0.094 & 0.025 & 0.356 & $<0.001$ \\
\hline
\end{tabular}

Two-sided Fisher's exact test was used to estimate differences between patients with peritoneal and non-peritoneal endometriosis

$N$ number of cases with relevant genotype, $p$ probability, $O R$ odds ratio, $95 \% C I$ confidence interval

endometriosis in KIR2DS5-positive, KIR2DS4del-negative persons was only $25 \%$ in comparison to $81 \%$ in the double-negative group. Similarly, KIR2DS5-negative, KIR2DS4del-positive, endometriotic persons had lower (almost 11 times) chance for peritoneal disease in relation to double negative ones $(\mathrm{OR}=0.094, P<0.001)$. Interestingly, although double positive (KIR2DS5 $\left.5^{+}, K_{R} 2 D S 4 d e l^{+}\right)$genotype had also a decreased chance of peritoneal endometriosis, in this case the protective effect was weaker (Table 2): this form of disease was present in nearly $43 \%$ of patients with this genotype. KIR2DS4-full-length gene presence was not related to severity of disease (data not shown). KIR2DS5 and KIR2DS4 genes are in a strong negative linkage disequilibrium, as reflected by a complete lack of haplotype positive both for KIR2DS5 and KIR2DS4del (more frequent variant of KIR2DS4) in the control group (Table 3). This haplotype is also absent from women with ovarian endometriosis, but present in $4 \%$ of patients with peritoneal form of disease (Table 3).

Last but not least, the severity of disease seemed to be associated with the KIR2DS4del gene, as the frequency of
KIR2DS4del was increased in higher disease stages (Table 4). No other genes had any effect on the severity of endometriosis (data not shown).

\section{Discussion}

Endometriosis is an enigmatic disease, in which many factors-genetic (Montgomery et al. 2008), neuroendocrine (Tariverdian et al. 2007), immunological (Tariverdian et al. 2007; Matarese et al. 2003) - are involved in the pathogenesis. The most accepted "transplantation theory" (Sampson 1927) explains the presence of the majority of endometriotic lesions in the peritoneal cavity by insufficient immune surveillance. Retrograde menstruation happens in up to $90 \%$ of women in reproductive age. However, only minority of these women develop endometriosis. Therefore, it is generally accepted that changes in the activity of immune cells in the peritoneal microenvironment play an important role in the etiology of this disease (Berkkanoglu and Arici 2003; Matarese et al. 2003; Dmowski and Braun 2004; Ulukus

Table 3 Linkage disequilibrium between two loci: KIR2DS4 and KIR2DS5

\begin{tabular}{|c|c|c|c|c|c|c|c|}
\hline Group & Haplotypes & 2DS4full/2DS5 & 2DS4del/2DS5 & $-/ 2 \mathrm{DS} 5$ & 2DS4full/- & 2DS4del/- & $-1-$ \\
\hline \multirow[t]{4}{*}{ Controls } & HFs & 0.0019 & 0 & 0.1724 & 0.153 & 0.6172 & 0.0555 \\
\hline & $r$ & -0.153 & -0.328 & 0.666 & 0.07 & 0.151 & -0.306 \\
\hline & $R^{2}=0.6952$ & & & \multirow{2}{*}{\multicolumn{4}{|c|}{$\chi^{2}=148.77 ; \mathrm{df}=2 ; P<0.00001$}} \\
\hline & $D_{\mathrm{KL}}=0.4701$ & & & & & & \\
\hline \multirow[t]{4}{*}{ Peritoneum+ } & HFs & 0 & 0.0422 & 0.0867 & 0.1976 & 0.5245 & 0.149 \\
\hline & $r$ & -0.16 & -0.114 & 0.323 & 0.061 & 0.044 & -0.124 \\
\hline & $R^{2}=0.1639$ & & & \multirow{2}{*}{\multicolumn{4}{|c|}{$\chi^{2}=9.178 ; \mathrm{df}=2 ; P=0.01016$}} \\
\hline & $D_{\mathrm{KL}}=0.1473$ & & & & & & \\
\hline \multirow[t]{3}{*}{ Peritoneum- } & HFs & 0.0044 & 0 & 0.1077 & 0.15 & 0.6707 & 0.0671 \\
\hline & $r$ & -0.098 & -0.274 & 0.629 & 0.035 & 0.097 & -0.224 \\
\hline & $\begin{array}{l}R^{2}=0.5413 \\
D_{\mathrm{KL}}=0.3093\end{array}$ & & & \multicolumn{4}{|c|}{$\chi^{2}=53.59 ; \mathrm{df}=2 ; P<0.00001$} \\
\hline
\end{tabular}

$H F s$ haplotype frequencies, $D_{K L}$ Kullback-Leibler divergence from linkage equilibrium, $R^{2}$ global squared correlation coefficient for two loci, $r$ correlation coefficient for allele frequencies, - lack of a given gene (KIR2DS4 and/or KIR2DS5), Peritoneum+ and Peritoneum-, the presence or absence of peritoneal endometriosis 
Table 4 Frequency of KIR2DS4del positivity depending on endometriosis stage

\begin{tabular}{lllc}
\hline Group & \multicolumn{2}{l}{ Disease stage } & \\
\cline { 2 - 4 } & I+II & III & IV \\
\hline 2DS4del- & 5 & 9 & 6 \\
2DS4del+ & 7 & 33 & 49 \\
$\%$ & 58.33 & 78.57 & 89.09 \\
$P$ & 0.012 & & \\
\hline
\end{tabular}

The chi-square test for trend was used to investigate the relationship between clinical and genetic variables

$p$ probability, $I+I I$ minimal and mild endometriosis, III moderate, $I V$ severe

et al. 2006). Particularly, it has been shown that NK cells of peripheral blood and peritoneal fluid of endometriotic patients exhibit decreased cytotoxicity to autologous endometrium (Oosterlynck et al. 1991; 1992). This impaired activity also correlated with severity of the disease. Moreover, Japanese scientists found an increased percentage of KIR2DL1positive NK cells in peritoneal fluid and peripheral blood of women with pelvic endometriosis, which suggests stronger inhibition of NK cell activity against ectopic endometrium (Maeda et al. 2002). Individual NK cells differ in their KIR repertoire because this results from stochastic KIR expression in individual clones (Parham 2005). Nevertheless, a high degree of genotypic polymorphism (i.e., the presence or absence of particular KIR genes in the genotype of a given individual) allows NK cells to express only those KIR genes which are present in their genotype. Therefore, we looked for possible associations of KIR genes with susceptibility to endometriosis, localization of lesions (ovarian versus peritoneal), and severity of the disease.

We previously described decreased frequency of KIR2DS5 in patients with endometriosis in comparison to healthy control (Nowak et al. 2010) but other KIR genes were not analyzed there. Here, we report the protective effect of KIR2DS5 only in females who possessed the HLA-C C2 group. The KIR2DS5 gene has been proven to be expressed at both the mRNA (Della Chiesa et al. 2008) and protein (Steiner et al. 2014) level, although it has been reported that its transcript could be detected in only $10 \%$ of individuals positively genotyped for this gene (Leung et al. 2005), and protein expression of different alleles varies (Steiner et al. 2014). However, analysis of KIR expression at the protein level is difficult because of cross-reactivity of available antibodies, especially between inhibitory and activating receptors, which frequently have very similar amino acid sequences in their extracellular domains. Recently, it has been shown that monoclonal antibodies 143211 and HPMA4, which were previously thought to be specific exclusively for KIR2DL1 and KIR2DS1/L1, respectively, also bind KIR2DS5 (143211) or KIR2DS5 and
KIR2DS3 (HPMA4) (Czaja et al. 2014). Therefore, studies showing increased expression of KIR2DL1 but not other KIRs in women with pelvic endometriosis (Maeda et al. 2002) should be treated with caution.

A physiologic ligand for KIR2DS5 is unknown (Parham et al. 2012). However, circumstantial evidence suggests that it may interact with both $\mathrm{C} 1$ and $\mathrm{C} 2$, because, in hematopoietic stem cell transplantation, leukemia-free survival and relapse rate depended on the presence or absence of the donor KIR2DS5 gene as well as on the $C 1 / C 2$ versus $C 1 / C 1$ or $C 2 /$ $C 2$ genotype of the recipient (van der Meer et al. 2008). Our finding, mentioned above, also suggests an interaction between KIR2DS5 and HLA-C molecules, influencing the ability of KIR2DS5 + NK cells to eliminate ectopic endometrium in $\mathrm{C} 2+$ women. Interestingly, KIR2DS5 was found protective against another gynecological disorder, pre-eclampsia, in British Europeans and Ugandan Africans, and fetal $C 2$ inherited from the father increased the risk of disease in KIR2DS5-negative women (Nakimuli et al. 2015). However, only one allele, $K I R 2 D S 5^{*} 006$, was protective in Ugandans, and this allele is absent from European populations, possessing only KIR2DS5*002 if any (Hou et al. 2009; Gonzalez-Galarza et al. 2015). Therefore, it might be that different KIR2DS5 alleles affect pre-eclampsia and endometriosis.

Moreover, we observed preferential non-peritoneal localization of lesions in patients possessing either the KIR2DS5 or KIR2DS4del gene, whereas simultaneous presence of both genes had a weaker effect. Interaction between these two genes has also been found recently in renal transplantation, where we detected a protective effect of the KIR2DS5 gene against acute graft rejection, but only in the absence of KIR2DS4del (Nowak et al. 2012). Interestingly, the effect of the KIR2DS4-full-length gene on the graft protection associated with KIR2DS5 was opposite, i.e., KIR2DS5 was protective only if KIR2DS4 full-length was present (Nowak et al. 2012). Here, we observed a much lower percentage of peritoneal endometriosis in women possessing either the KIR2DS5 or KIR2DS4del gene than when both were present. Moreover, even in the subgroup of KIR2DS5+/KIR2DS4del+ patients, the fraction with peritoneal lesions was twice as low as in women negative for both these genes. However, the numbers were too small to draw a firm conclusion.

KIR2DS5 and KIR2DS4 genes are in strong negative linkage disequilibrium in our population (Table 3 and Niepiekło-Miniewska et al. 2013), and KIR2DS4del and KIR2DS4-full-length are alleles. Therefore, individuals positive for KIR2DS5 and KIR2DS4del are highly unlikely to possess KIR2DS4-full-length; similarly, individuals positive for KIR2DS5 and KIR2DS4-full-length are highly unlikely to be KIR2DS4del-positive. In peritoneal endometriosis, we observed only a weak effect of KIR2DS4del on KIR2DS5 association with lack of endometrial lesions in the peritoneum, and no effect of KIR2DS4-full-length. 
KIR2DS4-full-length variant and KIR2DS5 had frequencies of only 18.3 and $11.5 \%$ in our patients, respectively, so the number of women with peritoneal endometriosis $(N=$ 55) was perhaps too small to detect the interaction of KIR2DS4-full-length with KIR2DS5 similar to that observed in kidney graft rejection.

Patients with endometriotic lesions limited to the peritoneum belong to a group of mild severity of this disease. NK cells may have the potential to remove endometriotic tissues from the peritoneal cavity but may not have such easy access to ovarian cysts, which are associated with more severe manifestation of endometriosis. Associations of KIR2S5 and KIR2DS4del with protection from peritoneal but not ovarian lesions in endometriotic patients suggest that peritoneal endometriosis may have a different genetic background than ovarian endometriosis. Indeed, the same KIR2DS4del which was associated with protection from peritoneal lesions was associated with the highest grades of endometriosis which encompass ovarian lesions.

The only report on KIR gene associations with endometriosis published so far in addition to our KIR2DS5 paper (Nowak et al. 2010) is that by Kitawaki et al. (2007), who described decreased frequency of KIR3DSI and increased frequency of highly inhibitory KIR-HLA combinations. This result is somewhat similar to ours, because KIR haplotypes consisting mostly of inhibitory KIR genes (called A haplotypes) are lacking KIR2DS5 although not KIR2DS4 (Jiang et al. 2012). In addition, we also observed slightly decreased presence of KIR 3DS1 in patients with endometriosis but it was not significant. The Japanese population demonstrates different KIR gene distribution from Europeans (allele frequency net: Gonzalez-Galarza et al. 2015), and is particularly rich in A haplotype homozygotes (Hiby et al. 2004).

In conclusion,

(i) The presence of KIR2DS5 reduced the risk of disease if a female possessed alleles from group $\mathrm{C} 2$ of $H L A-C$.

(ii) In endometriotic patients, KIR2DS5 or KIR2DS4del presence lowered the risk of localization of lesions in the peritoneum.

(iii) On the other hand, KIR2DS4del presence in patients was associated with severity of endometriosis, which, due to strong negative LD, may suggest an indirect effect of KIR2DS5 absence.

(iv) Our results suggest a different genetic background of peritoneal and ovarian endometriosis.

Zhang et al. (2006) proposed KIR and HLA expression studies as an immunodiagnostic parameter for pelvic endometriosis. We believe that our results may support their proposal and extend it to genotyping patients for KIR2DS4 and KIR2DS5, as well as for HLA-C C1 and $C 2$.
Acknowledgments The authors are grateful to patients and control volunteers for the donation of blood and their agreement to use their clinical data in this study. We are also grateful to Mr. Maciej Sobczynski, PhD., for his expert statistical analysis. This project was funded by grants no. 2.P05A.069.28 and N N401 588340 from the Ministry of Science and Higher Education, and by grants 14/2012 and 14/2013 from our institute.

Conflict of interest disclosure The authors declare that they have no conflict of interest.

Open Access This article is distributed under the terms of the Creative Commons Attribution License which permits any use, distribution, and reproduction in any medium, provided the original author(s) and the source are credited.

\section{References}

Abdallah JM, Goffinet B, Cierco-Ayrolles C, Perez-Enciso M (2003) Linkage disequilibrium fine mapping of quantitative trait loci: a simulation study. Genet Sel Evol 35:513-532

American Fertility Society (1985) Revised American Fertility Society classification of endometriosis. Fertil Steril 43:351-352

Berkkanoglu M, Arici A (2003) Immunology and endometriosis. Am J Reprod Immunol 50:48-59

Bhasi K, Zhang L, Brazeau D, Zhang A, Ramanathan M (2006) Information-theoretic identification of predictive SNPs and supervised visualization of genome-wide association studies. Nucleic Acids Res 34:e101

Burney RO, Giudice LC (2012) Pathogenesis and pathophysiology of endometriosis. Fertil Steril 98:511-519

Czaja K, Borer AS, Schmied L, Terszowski G, Stern M, Gonzalez A (2014) A comprehensive analysis of the binding of anti-KIR antibodies to activating KIRs. Genes Immun 15:33-37

Della Chiesa M, Romeo E, Falco M, Balsamo M, Augugliaro R, Moretta L (2008) Evidence that the KIR2DS5 gene codes for a surface receptor triggering natural killer cell function. Eur J Immunol 38: 2284-2289

Dmowski WP, Braun DP (2004) Immunology of endometriosis. Best Pract Res Clin Obstet Gynaecol 18:245-263

Dunselman GAJ, Vermeulen N, Becker C et al (2014) ESHRE guideline: management of women with endometriosis. Hum Reprod 29:400 412

Excoffier L, Slatkin M (1995) Maximum-likelihood estimation of molecular haplotype frequencies in a diploid population. Mol Biol Evol 12:921-927

Falco M, Moretta L, Moretta A, Bottino C (2013) KIR and KIR ligand polymorphism: a new area for clinical applications? Tissue Antigens $82: 363-373$

Frohn C, Schlenke P, Ebel B, Dannenberg C, Bein G, Kitchner H (1998) DNA typing for natural killer cell inhibiting HLA-Cw groups NK1 and NK2 by PCR-SSP. J Immunol Methods 218:155-160

Gonzalez-Galarza FF, Christmas S, Middleton D, Jones AR (2015) Allele frequency net: a database and online repository for immune gene frequencies in worldwide populations. Nucleic Acids Res 43(Database issue):D784-D788

Graef T, Moesta AK, Norman PJ, Abi-Rached L, Vago L, Older Aguilar AM (2009) KIR2DS4 is a product of gene conversion with KIR3DL2 that introduced specificity for HLA-A*11 while diminishing avidity for HLA-C. J Exp Med 206:2557-2572 
Gupta S, Goldberg JM, Aziz N, Goldberg E, Krajcir N, Agarwal A (2008) Pathogenic mechanisms in endometriosis-associated infertility. Fertil Steril 90:247-257

Hiby SE, Walker JJ, O’Shaughnessy KM, Redman CW, Carrington M, Trowsdale J (2004) Combinations of maternal KIR and fetal HLA-C genes influence the risk of preeclampsia and reproductive success. $\mathrm{J}$ Exp Med 200:957-965

Hou L, Chen M, Jiang B, Kariyawasam K, Ng J, Hurley CK (2009) In contrast to other stimulatory natural killer cell immunoglobulin-like receptor loci, several KIR2DS5 alleles predominate in African Americans. Hum Immunol 70:733-737

Jiang J, Johnson C, Jayaraman J, Simecek N, Noble J, Moffatt MF (2012) Copy number variation leads to considerable diversity for B but not A haplotypes of the human KIR genes encoding NK cell receptors. Genome Res 22:1845-1854

Kitawaki J, Xu B, Ishihara H, Fukui M, Hasegawa G, Nakamura N (2007) Association of killer cell immunoglobulin-like receptor genotypes with susceptibility to endometriosis. Am J Reprod Immunol $58: 481-486$

Kulkarni S, Martin MP, Carrington M (2008) The Yin and Yang of HLA and KIR in human disease. Semin Immunol 20:343-352

Leung W, Iyengar R, Triplett B, Turner V, Behm FG, Holladay MS (2005) Comparison of killer Ig-like receptor genotyping and phenotyping for selection of allogeneic blood stem cell donors. J Immunol 174:6540-6545

Liu Z, Lin S (2005) Multilocus LD measure and tagging SNP selection with generalized mutual information. Genet Epidemiol 29:353-364

Maeda N, Izumiya C, Yamamoto Y, Oguri H, Kusume T, Fukaya T (2002) Increased killer inhibitory receptor KIR2DL1 expression among natural killer cells in women with pelvic endometriosis. Fertil Steril 77:297-302

Matarese G, De Placido G, Nikas Y, Alviggi C (2003) Pathogenesis of endometriosis: natural immunity dysfunction or autoimmune disease? Trends Mol Med 9:223-228

Middleton D, Gonzelez F (2010) The extensive polymorphism of KIR genes. Immunology 129:8-19

Montgomery GW, Nyholt DR, Zhao ZZ, Treloar SA, Painter JN, Missmer SA (2008) The search for genes contributing to endometriosis risk. Hum Reprod Update 14:447-457

Nakimuli A, Chazara O, Hiby SE et al (2015) A KIR B centromeric region present in Africans but not Europeans protects pregnant women from pre-eclampsia. PNAS 112:845-850

Niepiekło-Miniewska W, Majorczyk E, Matusiak $Ł$ et al (2013) Protective effect of the KIR2DS1 gene in atopic dermatitis. Gene 527:594-600

Nowak I, Malinowski A, Tchórzewski H et al (2009) Frequencies of killer immunoglobulin-like receptor genotypes influence susceptibility to spontaneous abortion. J Appl Genet 50:391-398
Nowak I, Majorczyk E, Wiśniewski A et al (2010) Does the KIR2DS5 gene protect from some human diseases? PLoS One 5:e12381

Nowak I, Magott-Procelewska M, Kowal A et al (2012) Killer immunoglobulin-like receptor (KIR) and HLA genotypes affect the outcome of allogeneic kidney transplantation. PLoS One 7: e44718

Oosterlynck DJ, Cornillie FJ, Waer M, Vandeputte M, Koninckx PR (1991) Women with endometriosis show a defect in natural killer activity resulting in a decreased cytotoxicity to autologous endometrium. Fertil Steril 56:45-51

Oosterlynck DJ, Meuleman C, Waer M, Koninckx PR (1992) The natural killer activity of peritoneal fluid lymphocytes is decreased in women with endometriosis. Fertil Steril 58:290-295

Parham P (2005) MHC class I molecules and KIRs in human history, health and survival. Nat Rev Immunol 5:201-214

Parham P, Norman PJ, Abi-Rached L, Guethlein LA (2012) Humanspecific evolution of killer cell immunoglobulin-like receptor recognition of major histocompatibility complex class I molecules. Philos Trans R Soc Lond B Biol Sci 19(367):800-811

Sampson J (1927) Peritoneal endometriosis due to menstrual dissemination of endometrial tissue into the peritoneal cavity. Am J Obstet Gynecol 14:422-467

Steiner NK, Dakshanamurthy S, Nguyen N, Hurley CK (2014) Allelic variation of killer cell immunoglobulin-like receptor 2DS5 impacts glycosylation altering cell surface expression levels. Hum Immunol 75:124-128

Sun JY, Gaidulis L, Miller MM, Goto RM, Rodriguez R, Forman SJ, Senitzer D (2004) Development of a multiplex PCR-SSP method for killer cell immunoglobulin-like receptor genotyping. Tissue Antigens 64:462-468

Tariverdian N, Theoharides TC, Siedentopf F, Gutiérrez G, Jeschke U, Rabinovich GA (2007) Neuroendocrine-immune disequilibrium and endometriosis: an interdisciplinary approach. Semin Immunopathol 29:193-210

The Practice Committee of the American Society for Reproductive Medicine (2012) Endometriosis and infertility: a committee opinion. Fertil Steril 98:591-598

Ulukus M, Cakmak H, Arici A (2006) The role of endometrium in endometriosis. J Soc Gynecol Investig 13:467-476

Van der Meer A, Schaap NPM, Schattenberg A, van Cranenbroek B, Tijssen HJ, Joosten I (2008) KIR2DS5 is associated with leukemia free survival after HLA identical stem cell transplantation in chronic myeloid leukemia patients. Mol Immunol 45:3631-3638

Zhang C, Maeda N, Izumiya C, Yamamoto Y, Kusume T, Oguri H (2006) Killer immunoglobulin-like receptor and human leukocyte antigen expression as immunodiagnostic parameters for pelvic endometriosis. Am J Reprod Immunol 55:106-114 